\title{
Simultaneous quantification of myocardial and blood flow velocities based on duplex mode ultrasound imaging
}

\author{
Christer Grönlund ${ }^{1,2^{*}}$, Kenji Claesson ${ }^{1}$, Jan D'hooge ${ }^{3}$, Michael Y Henein ${ }^{4}$ and Per Lindqvist ${ }^{4}$
}

\author{
* Correspondence: christer. \\ gronlund@vll.se \\ 'Department of Biomedical \\ Engineering - R\&D, Radiation \\ Sciences, Umeå University, Umeå \\ 90185, Sweden \\ ${ }^{2}$ Centre for Biomedical Engineering \\ and Physics (CMTF), Umeå \\ University, Umeå, Sweden \\ Full list of author information is \\ available at the end of the article
}

\begin{abstract}
Background: Ultrasound imaging of the heart is a commonly used clinical tool to assess cardiac function. The basis for this analysis is the quantification of cardiac blood flow and myocardial velocities. These are typically measured using different imaging modes and on different cardiac cycles. However, due to beat-to-beat variations such as irregular heart rhythm and transient events, simultaneous acquisition is preferred. There exists specialized ultrasound systems for this purpose; however, it would be beneficial if this could be achieved using conventional ultrasound systems due to their wide availability. The conventional Duplex mode ultrasound allows simultaneous acquisition, however at a highly reduced spatial and temporal resolution.

Methods: The aim of this work was to present and evaluate the performance of a novel method to recover myocardial tissue velocity using conventional Duplex ultrasound imaging, and to demonstrate its feasibility for the assessment of simultaneous blood flow and myocardial velocity in-vivo. The essence of the method was the estimation of the axial phase shift of robust echogenic structures between subsequent image frames. The performance of the method was evaluated on synthetic tissue mimicking B-mode image sequences at different frame rates $(20-60 \mathrm{~Hz})$ and tissue velocities (peak velocities $5-15 \mathrm{~cm} / \mathrm{s}$ ), using cardiac deformation and displacement characteristics. The performance was also compared to a standard 2-D speckle tracking technique.
\end{abstract}

Results: The method had an overall high performance at frame rates above $25 \mathrm{~Hz}$, with less than 15\% error of the peak diastolic velocity, and less than 10 ms peak timing error. The method showed superior performance compared to the 2-D tracking technique at frame rates below $50 \mathrm{~Hz}$. The in-vivo quantification of simultaneous blood flow and myocardial tissue velocities verified the echocardiographic patterns and features of healthy subjects and the specific patient group.

Conclusions: A novel myocardial velocity quantification method was presented and high performance at frame rates above $25 \mathrm{~Hz}$ was shown. In-vivo quantification of simultaneous myocardial and blood flow velocities was feasible using the proposed method and conventional Duplex mode imaging. We propose that the methodology is suitable for retrospective as well as prospective studies on the mechanics and hemodynamics of the heart.

Keywords: Ultrasound, Heart, Simultaneous, Echocardiography, Blood flow, Myocardial, Velocity, Low frame rate, Duplex

\section{Biomed Central}




\section{Background}

\section{Cardiac ultrasound and beat-to-beat variations}

Ultrasound imaging of the heart is a commonly used clinical tool to assess cardiac function. The basis for this analysis is the quantification of cardiac blood flow velocities and myocardial motion. Typically, they are consecutively acquired using separate ultrasound imaging modes, i.e., on different cardiac beats. Thus, they are not measured in a simultaneous fashion, and beat-to-beat variations such as physiological transients (e.g., stress), respiration (load), and heart rate variability is not accounted for [1]. In particular, diagnostic assessment of patients with irregular heart rhythms, atrial fibrillation, and diastolic dysfunction would improve from a simultaneous measurement [2].

\section{Simultaneous myocardial and blood flow velocity}

Recently, techniques for simultaneous acquisition of myocardial motion and blood flow velocity were demonstrated. However, they were based on specialized ultrasound scanner systems with customized imaging modes $[2,3]$. Thus these techniques are not available to the vast majority of ultrasound users.

On conventional clinical ultrasound systems the Duplex imaging mode achieves a simultaneous acquisition of myocardial tissue and blood flow velocity (Color Doppler mode, CDI). Here, the Doppler mode images are interleaved with B-mode images, and colourcoded on top of the greyscale images. This results in relatively low frame rates (typically 20-40 Hz) of the B-mode sequence; there is a trade-off between CDI and B-mode frame rates (typical setting is 3:1 between CDI frame rate and B-mode frame rate). In addition, the B-mode images comprise less image lines compared to standard B-mode, resulting in a crude 2-D representation of the tissue structures (lower lateral spatial resolution).

Due to the low frame rate and low lateral spatial resolution the speckle pattern decorrelation is very high [4], and the relative lateral and out-of-plane motion of the heart will be significant. As a consequence, traditional tissue velocity estimation using speckle tracking methods $[5,6]$ performs poorly and dedicated software may not allow the estimation of myocardial velocities.

Aim

The aim of this work was to 1) present and evaluate the performance of a novel method to recover myocardial tissue velocity using conventional duplex ultrasound imaging, and to 2) exemplify its feasibility for the assessment of simultaneous blood flow and myocardial velocity in-vivo.

\section{Methods}

\section{Myocardial velocity quantification}

The method to recover the axial tissue velocity was based on five steps (block scheme of Figure 1A). Initially, a 2-D region-of-interest (ROI) was selected in the image. Next, the 2-D B-mode images were cropped to the size of the ROI (Figure 1B). The cropped image sequence will here be denoted $I(y, x, t)$, where $y$ is depth (axial), $x$ is width (lateral), and $t$ is time. Subsequently, the 2-D images were reduced to $1-\mathrm{D}$ representations by taking the laterally most echogenic structures (Figure $1 \mathrm{~B}$ ) as:

$$
I^{\prime}(y, t)=\max _{x} I(y, x, t) .
$$




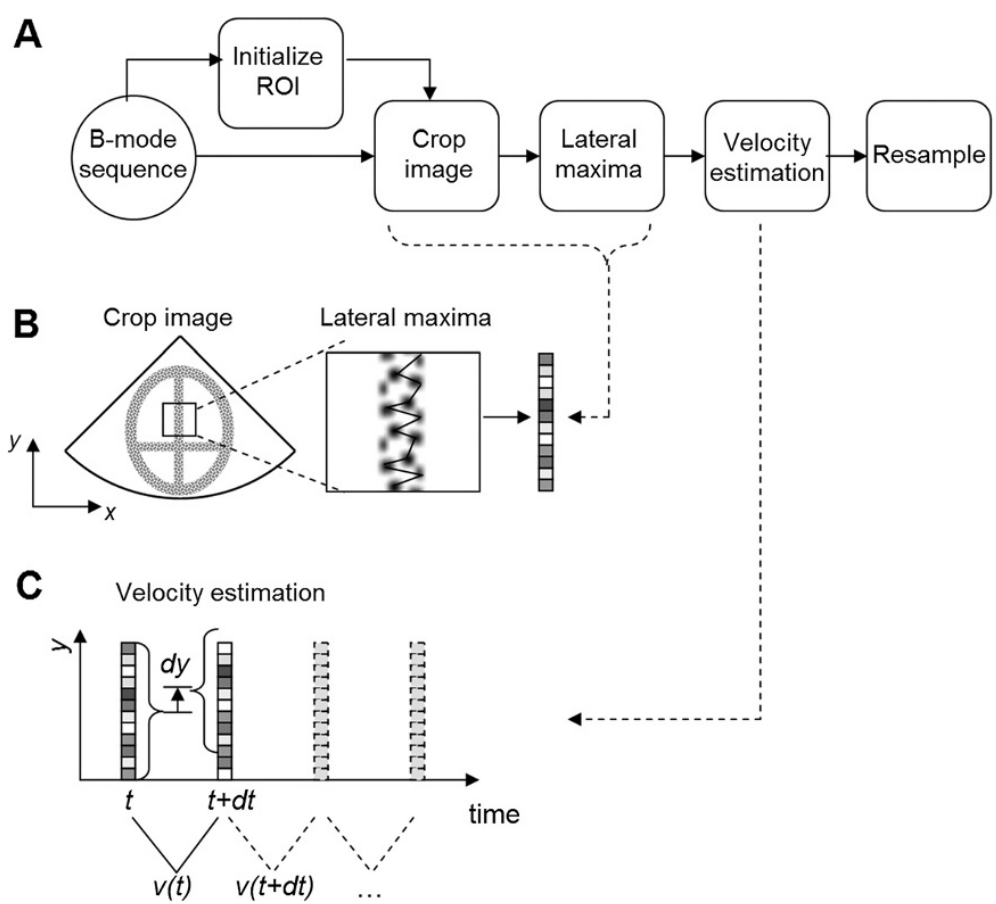

Figure 1 Illustration of the myocardial tissue velocity quantification procedure. (A) Block scheme of the procedure including five steps: Initially. a (ROI) was selected. Subsequently, the ROI was used for cropping the images in the sequence, prior to reduction of the 2D B-mode images' echo-pattern to 1D projections. The tissue velocity was estimated using spatial phase shift and finally the velocity signal was resampled to increase temporal resolution. (B) Illustration of ROI selection, cropped image and reduction of image to 1D by taking the lateral echogenic maxima. (C) Myocardial velocity was estimated based on the spatial phase shift of the 1D projections between pair-wise consecutive frames. Color of schematic ultrasound images are inverted, where dark area corresponds to an echogenic region.

The motivation for this reduction was that the tissue has a rather poor 2-D image representation due to relatively few image lines. In addition, the most highly reflective pixels have the best signal-to-noise ratio (SNR), and represent larger structures (and not speckle-pattern), thus more likely to be visible between frames at low frame rates and thus more robust to out-of-plane motion.

The axial tissue velocity was estimated based on the spatial phase-shift of pairwise consecutive 1-D tissue profiles (Figure 1C). The spatial phase-shift was calculated using the lag, $n_{y}$ of the maximum of the cross-correlation function, $R$, as

$$
n_{y}(t)=\underset{y}{\operatorname{argmax}} R\left(I^{\prime}(y, t+d t), I^{\prime}(y, t)\right) \text {. }
$$

The corresponding tissue velocity was then calculated as

$$
v(t)=n_{y}(t) \cdot \frac{d y}{d t},
$$

where $d y$ is the axial resolution of the images. To increase the resolution of the estimated velocity, the spatial dimension of the 1-D signals, $I^{\prime}(y, t)$, were up-sampled by a factor of 20 prior to the cross-correlation calculation. 
Finally, the tissue velocity signal, $v(t)$, was resampled at $1000 \mathrm{~Hz}$ (from the original 20-60 Hz) using interpolation (cubic splines) in order improve the waveform resemblance and allow estimation of timing of peaks.

\section{Evaluation of performance of myocardial velocity quantification}

The performance of the method was evaluated using synthetic B-mode septum tissue mimicking image sequences (see Appendix A for calculation details). Matlab code (version 2011b, Mathworks, Nattick, MA, USA) for generating the data can be downloaded at www.vll.se/mt/fou/). In essence, synthetic B-mode image sector scans with 64 lines at 45 degrees field-of-view were generated by sampling image planes of a synthetic 3-D tissue volume (2-D image section shown in Figure 2A) after imposing lateral and outof-plane motion displacement of the tissue. In addition, the axial displacement was achieved by deforming the synthetic volume in the axial direction. The tissue motion was based on authentic myocardial tissue velocity waveforms (Figure 2B). Ninety-six cardiac-cycles were simulated for combinations of frame rates between 20 to $60 \mathrm{~Hz}$, and diastolic peak velocities at 5, 10 and $15 \mathrm{~cm} / \mathrm{s}$. The cardiac cycle tissue waveform for each simulation was randomly selected out of three different ones (Figure 1B). The resulting axial strain was about 6,12 , and $18 \%$ for 5,10 and $15 \mathrm{~cm} / \mathrm{s}$ velocities, respectively.

The performance of the proposed method was assessed using A) the estimation error between the true peak and the estimated peak velocity (E'), B) the timing error between the true and estimated peaks, and C) the cross-correlation value at the E' (as a measure of estimation quality). See Figure 3A for illustration of measures of performance.

In addition, the performance of the proposed method was compared to a standard 2-D speckle-tracking technique. Two-dimensional cross-correlation was used to calculate the axial spatial lag between pair wise consecutive frames of the cropped image sequence,

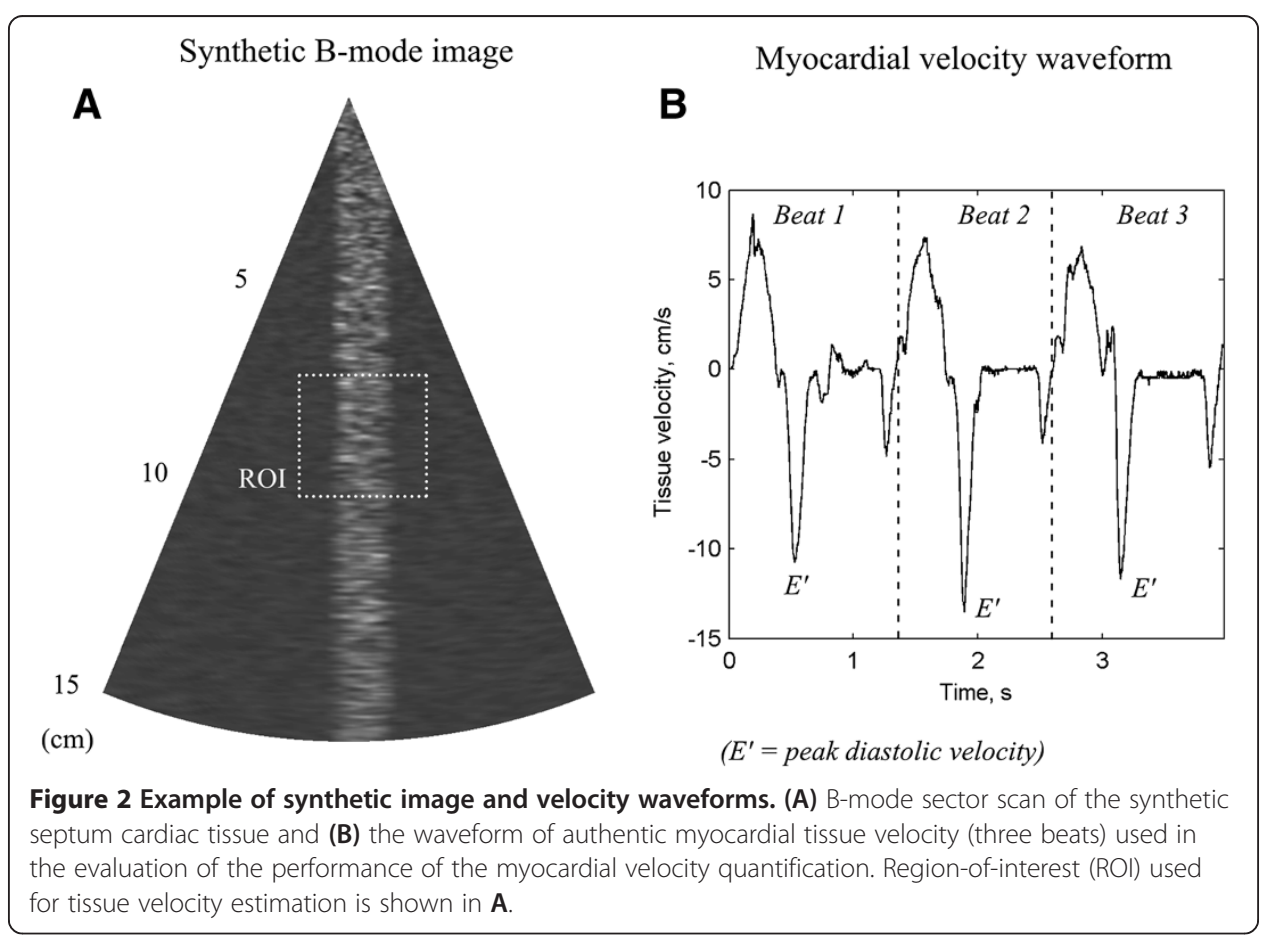




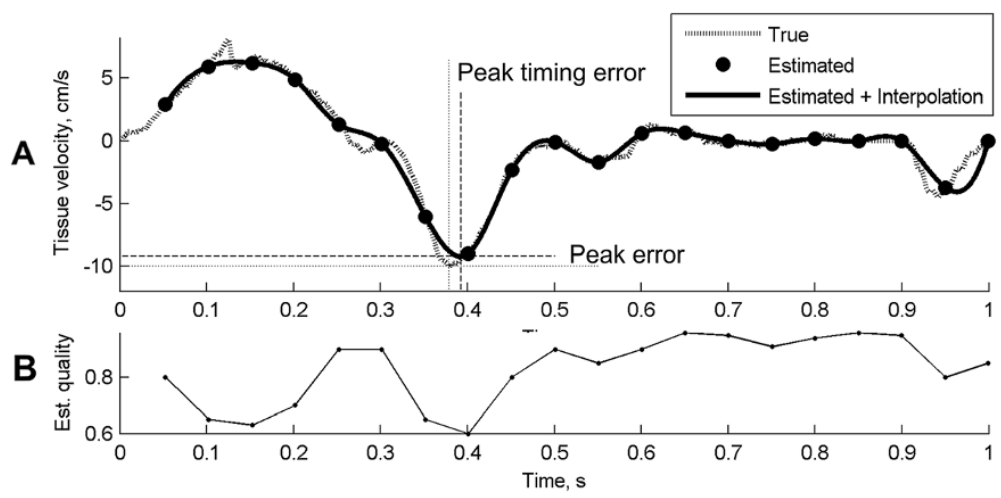

Figure 3 Measures of performance. (A) Example of estimated and true myocardial velocity curves from a typical simulation of one cardiac cycle at $20 \mathrm{~Hz}$ frame rate. Peak error and peak timing error was calculated as the difference between the true waveform peak amplitude and position, respectively. The velocity estimation quality (cross-correlation peak) is illustrated in $\mathbf{B}$.

$I(y, x, t)$. Prior to the 2-D cross-correlation calculation, the axial resolution of the images were up-sampled by a factor 20 , and the final tissue velocity signal was resampled at $1000 \mathrm{~Hz}$ using interpolation (cubic splines). Thus, the velocity estimation resolution and temporal resolution was comparable as with the proposed method.

The methods were compared using two separate sets of simulated signals. The first set featured pure axial displacement of tissue, i.e., no out-of-plane motion and no deformation (so called "best-case" conditions). The second set featured axial deformation as well as out-of-plane motion (so called "worst-case" conditions).

\section{In-vivo duplex mode acquisition}

Two subjects participated in this work; one healthy subject, age 40 years, with no medical history of cardiac disease, and one patient with cardiac disease: biopsy-proved hereditary transthyretin amyloidosis, age 45 . Each participant gave a written consent prior to the tests. The test conformed to the declaration of Helsinki and was accepted by the local ethics committee of Umeå University, Sweden.

Ultrasound image sequences where acquired using a Vivid 7 ultrasound scanner with a M4S cardiac probe (GE Medical, Horten, Norway) in Duplex mode (B/CDI). Sector depth, width and location were optimized for maximal frame rate, and allowing simultaneous registration of motion of the septal myocardial wall and the blood flow velocity across the mitral annulus. The frame rate of the CDI and $\mathrm{B}$ images were about $100 \mathrm{~Hz}$ and $33 \mathrm{~Hz}$, respectively. Image sequences were exported in hdf5 format, with B-mode and CDI-modes as separate image sequences at 8-bit resolution, for offline processing.

\section{Blood flow velocity quantification}

First, the CDI images of the duplex mode where filtered using a $3 \times 3$ pixel 2-D median filter. Next, the mitral blood flow velocity was extracted from a ROI covering the tip of the open mitral valves. The distribution of blood flow velocities from within this ROI was visualized as a spectral representation including all frames of the entire cardiac beat. 


\section{Results}

\section{Performance of myocardial velocity quantification}

Figure 3 presents the estimated and true myocardial velocity curves from a typical simulation of one cardiac cycle. In particular, the improvement of the interpolation on the sampled velocity curve can be seen in Figure 3A, in terms of both peak velocity accuracy and its timing as compared to the true velocity curve.

The results of the performance evaluation are presented in Figure 4A-B. In general, the performance increased with increasing frame rate and decreasing tissue velocity. When the frame rate was above $25 \mathrm{~Hz}$, the peak velocity error was on average less than $-15 \%$ (Figure $4 \mathrm{~A}$ ), and the peak timing error was less than $10 \mathrm{~ms}$ (Figure 4B). It should be stressed that the theoretical average timing error at $1 /(2 *$ frame rate) without waveform interpolation is much higher (illustrated by dotted line in Figure 4B). The estimation quality (cross-correlation values at the estimated peak) increased with increasing frame rate, was in the range 0.5 to 0.9 , and was higher than 0.75 for frame rates above $25 \mathrm{~Hz}$.
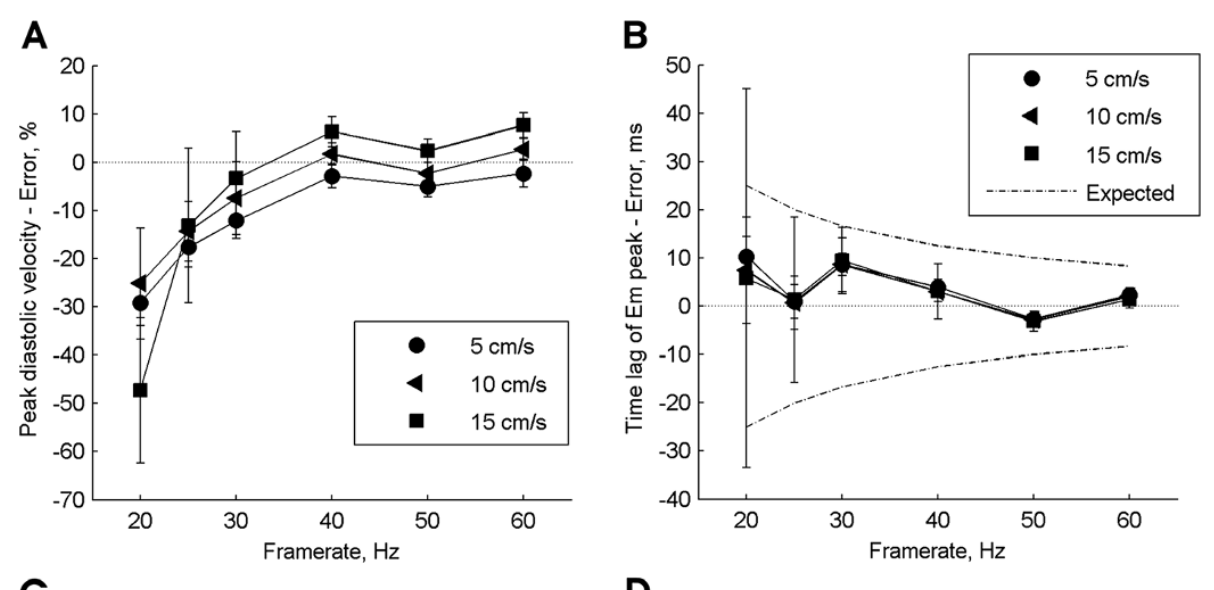

\section{C}

\section{D}
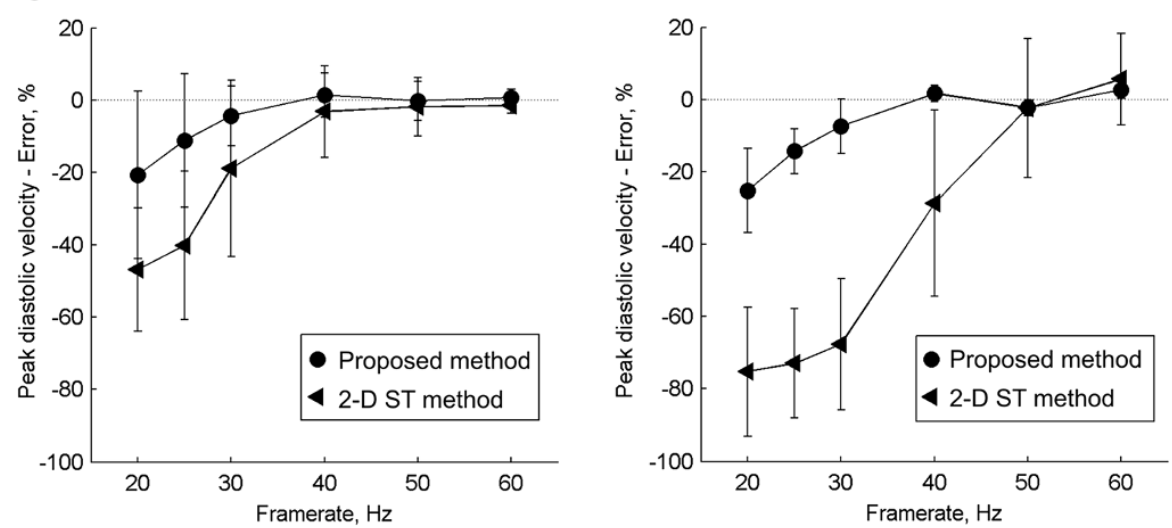

Figure 4 Performance of myocardial velocity quantification method. A) The peak error was calculated as the relative difference between the true and estimated velocities. B) Peak timing error was the time difference between the true peak position and the estimated peak position. The theoretical limit (dotted line) is half the inverse of frame rate and is the upper limit for the non-interpolated timing error. C) Comparison of performance between the proposed method and 2-D speckle tracking method in mild "best case" conditions (no lateral or out-of-plane motion, and only axial displacement of tissue). D) Same as in C but in rough "worst case" conditions (including axial deformation, and out-of-plane motion). 
Figure 4C and 4D present the comparison between the proposed method and the 2D speckle tracking technique for simulated signals with $10 \mathrm{~cm} / \mathrm{s}$ peak velocity. For the mild conditions the proposed method demonstrated lower error than the 2-D method for frame rates below $40 \mathrm{~Hz}$, and similar performance above $40 \mathrm{~Hz}$ (Figure 4C). For the rough conditions the proposed method had significantly higher lower error compared to the 2-D technique below frame rates of $50 \mathrm{~Hz}$.

\section{In-vivo simultaneous blood flow and myocardial velocity}

Figure 5 shows an image from the Duplex mode ultrasound sector scan and its overlaid B-mode and CDI images for the healthy subject. The image was taken during the diastolic filling phase of the heart. Figure 6 demonstrates results from one cardiac cycle on simultaneous mitral blood flow velocity (top), estimated septum myocardial velocity (middle), and corresponding electrocardiogram (bottom). A clear difference in filling patterns was observed between the healthy subject and the patient. For the healthy subject the peak myocardial velocity preceded the peak blood flow in the diastolic phase, with $\mathrm{E}^{\prime} \mathrm{E}^{\prime} \approx 5$, and $\mathrm{E} / \mathrm{A} \approx 1.4$. For the patient, the peak myocardial and blood flow velocities were synchronous, $\mathrm{E} / \mathrm{E}^{\prime} \approx 15, \mathrm{E} / \mathrm{A} \approx 1.0$, and the myocardial velocity was much lower than for the healthy subject.

\section{Discussion}

This work presents a novel method for myocardial velocity quantification. The performance of the method was evaluated on image sequences at low frame rates, common with B-mode image sequences obtained from conventional Duplex mode

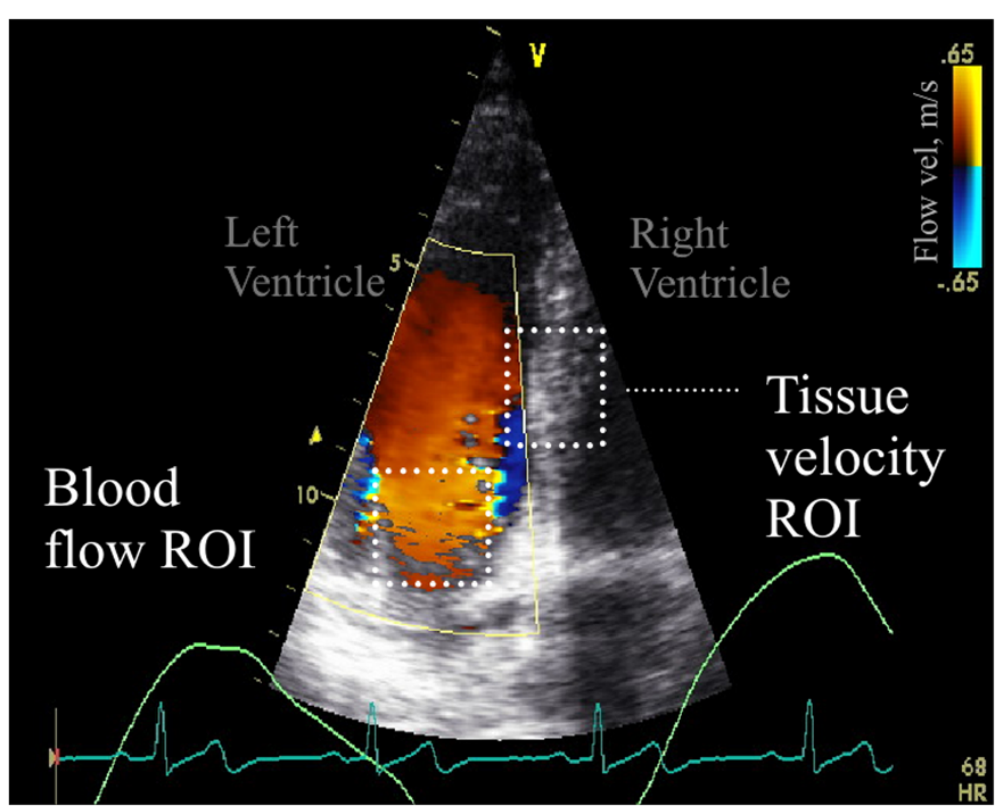

Figure 5 Example of in-vivo Duplex mode ultrasound imaging of the heart from a healthy subject. Image shows the filling of the left ventricle during diastole (red color is upward flow). The blood flow velocity (CDI mode) is color-coded on top of the grayscale tissue image (B-mode). The rectangular ROls indicate the regions used for myocardial tissue velocity, and mitral blood flow velocity quantification. The frame rates of the CDI and B-mode image sequences were 100 and $33 \mathrm{~Hz}$ respectively. The ECG and respiration signals are visible at the bottom of the figure. 

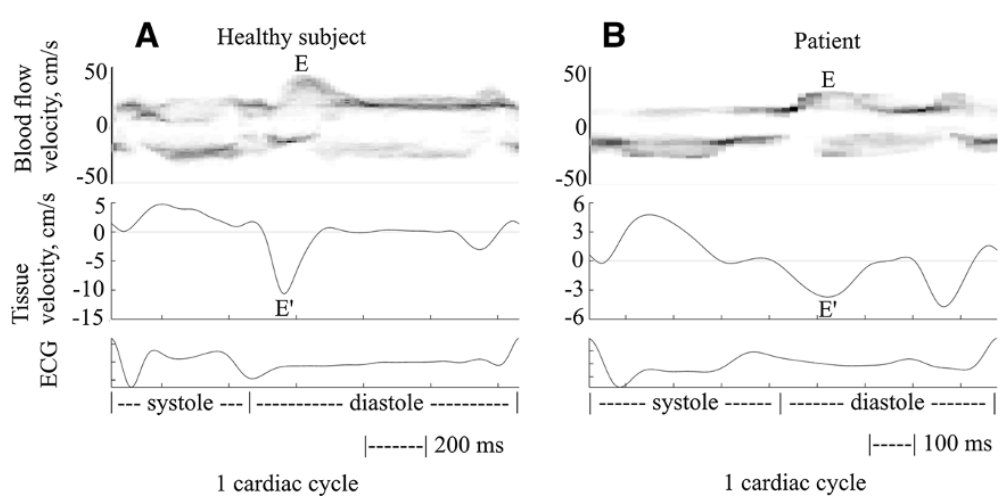

Figure 6 Examples of quantification of simultaneous blood flow velocity (top) and myocardial motion (middle) during one cardiac cycle. The corresponding electrocardiogram (ECG) can be seen in the bottom figure. The peak diastolic velocities are indicated as E and $E^{\prime}$ for blood flow and myocardial tissue respectively. The peak of the tissue velocity preceded the peak of the blood flow in the healthy subject (left side), while the patient presented syncronized peaks (right side). The healthy subject also had higher velocities than the patient. These findings were expected.

ultrasound imaging. In addition, in-vivo simultaneous quantification of blood flow and myocardial tissue velocity was demonstrated.

\section{Myocardial velocity quantification}

The performance of the proposed method shows that the performance was high at frame rates above $25 \mathrm{~Hz}$, with a peak velocity error of less than $15 \%$ and low timing error (less than $10 \mathrm{~ms}$ ). It should be stressed that the performance was evaluated on simulations of myocardial axial deformation in addition to both lateral and out of plane motion, and thus relatively rough conditions. The low timing error is likely attributed to the interpolation step of the velocity signal, and was also much lower than the theoretical frame rate limited error of $25 \mathrm{~ms}$ (at $25 \mathrm{~Hz}$ ).

The performance of the proposed method was comparable with the 2-D speckle tracking method under the mild conditions (best-case). However, for the rough conditions, the proposed method was superior to the 2-D method below frame rates of 50 Hz. This demonstrates the high robustness of the method at the low frame rates.

The peak velocity error was lower than, and timing error similar to what was found by Mårtensson et al. [7] studying the inter-system variations of tissue velocity imaging mode (TVI). They used a mechanical tissue phantom with pure axial displacement and found an error of $12.6 \%$ (range $0-34 \%$ ) on myocardial peak diastolic velocity, and a timing error of $2.9 \mathrm{~ms}$ (range 0.6-10.7 ms). It should be stressed that they investigated much higher frame rates, between $92-168 \mathrm{~Hz}$, as compared to ours at about $30 \mathrm{~Hz}$.

The performance of the method was evaluated on synthetic B-mode image sequences derived using an in-house developed software. While some other studies using simulated ultrasound images have used the Field II simulation program [8], we did not use this due to the much higher computational complexity. Given the number of simulations required and degrees of freedom included in the myocardial motion and deformation, a comprehensive software for this purpose was developed. The myocardial tissue texture of the simulated B-mode images resembled the in-vivo images and also images from synthetic phantoms as used in [7]. 


\section{Simultaneous blood flow velocity and myocardial velocity}

The results of the simultaneous blood flow and myocardial velocity from the two subjects were in accordance with what was expected: The myocardial motion preceded the mitral flow in the diastolic filling phase (blood is sucked into the ventricle due to a pressure gradient) in the healthy subject (Figure 6A). In the patient, there was no visible time lag between the blood flow and myocardial motion (Figure 6B) which is also typical for this group of patients. The indices of diastolic function (E/A, and $\left.E / E^{\prime}\right)$ were similar to those reported in the literature for both subjects. Taken together the quantification of simultaneous myocardial motion and blood flow velocity was feasible based on Duplex mode imaging of a healthy and a patient.

One of the main goals in echocardiographic procedures is the estimation of cardiac pressures and pressure gradients (filling and relaxation pressures within the cavities of the heart). In particular, the ratio between the blood flow peak early diastolic velocities and myocardial early velocities (i.e., E/E') is strongly correlated with the left ventricular filling pressure (LVFP) [9]. Li et al. [2] showed that this correlation is significantly improved when simultaneous E and E' is used. Recently our group found that the temporal difference between isovolumic relaxation times (IVRT) measured from blood flow and myocardial motion (non simultaneous measurement) of early diastole was strongly correlated with LVFP [10]. Based on the low timing error of the proposed technique in the present work, a interesting future study is to study the relation between the IVRT and filling pressure when blood flow and myocardial velocity is measured in a simultaneous fashion.

The assessment of mitral flow velocity was based on the distribution of velocities within a ROI, which is similar to the typical representation in echocardiography [11]. However, it should be noted that the relatively low frame rate of blood flow velocity acquisition is known to cause lower velocities [12].

An important application of the proposed methodology could be in retrospective studies of simultaneous blood flow velocity and myocardial velocities analysing ultrasound scan stored in databases. In addition, the method may be applied for the study of variations in echocardiographic variables and indices over multiple cardiac cycles, due to the inherent motion robustness and relatively low computational complexity. Currently the authors are pursuing such studies to further investigate the coordination between blood flow velocity and myocardial motion in healthy subjects and in patients.

\section{Conclusions}

This work presents a novel method for myocardial velocity quantification. The method demonstrated strong performance at frame rates above $25 \mathrm{~Hz}$ with a peak velocity error of less than $15 \%$, and a peak timing error of less than $10 \mathrm{~ms}$. In addition, the method was superior to a traditional 2-D tracking method at frame rates below $50 \mathrm{~Hz}$. In-vivo simultaneous quantification of blood flow and myocardial velocity was demonstrated based on conventional Duplex-mode ultrasound imaging and the proposed method. Simultaneous assessment of blood flow and myocardial velocity may allow detailed studies of the interaction between mechanical and hemodynamical processes within the heart. We propose that the method can be applied in both prospective as well as retrospective studies. 


\section{Appendix A}

\section{Synthetic tissue B-mode image sequence generation}

Synthetic tissue echo mimicking pattern volumes were generated based on 3-D white noise, and each pixel value was taken as its $4^{\text {th }}$ power before the volume was 3-D lowpass filtered. The lateral resolution was decreased with increased depth. The parameters were set similar to those obtained using Duplex mode on the Vivid 7 (GE Medical, Horten, Norway), at 45 degrees field-of-view (FOV), resulting in a 3:1 ratio in frame rate between CDI and B-mode. The scan depth was $15 \mathrm{~cm}$ and the axial resolution was $0.2 \mathrm{~mm} /$ pixel. Synthetic B-mode image sector scans with 64 lines at 45 degrees FOV were generated by sampling image planes of the synthetic tissue volume at time stamps corresponding to a given frame rate (Figure 2A). Line-by-line acquisition was used to sample the sector scan lines. To simulate lateral and out-of-plane motion the 3-D tissue volume was displaced in the lateral and the out-of-plane direction, respectively, during the cardiac cycle, throughout the volume $(5 \mathrm{~mm}$ peak displacement for both, with displacement waveform taken the same as the axial tissue velocity curve). The septum tissue was positioned in the center of the images with a $0.75 \mathrm{~mm}$ thickness, and left and right ventricles were set to zero intensity. This resulted in a realistic dynamic range. Finally, the B-mode scans were converted to 8-bit resolution. The axial tissue motion waveform was taken from a typical tissue velocity measurement [7] (Figure 2B), and was generated by compressing the axial tissue pattern, and lateral and out-of-plane tissue motion was generated by interpolating the 3-D tissue image according to the corresponding displacement at the time stamps. A random variation in temporal onset of the waveform was imposed as 0.5 /framerate variation, and Gaussian white noise (10 dB, peak-to-peak) was added to the images.

\section{Abbreviations}

ROI: Region-of-interest; B-mode: Brightness mode; CDI: Color doppler imaging; LVFP: Left-ventricular filling pressure; TVI: Tissue velocity imaging; SNR: Signal-to-noise ratio; FOV: Field-of-view.

\section{Competing interests}

The authors declare that they have no competing interests.

\section{Authors' contributions}

CG: composed the manuscript, CG and KC: analysed the data and worked on the methods, CG and PL: proposed the idea, $C G, J H, M H, P L$ : made the discussions. All authors read and approved the final manuscript.

\section{Acknowledgements}

The authors thank MSc. Ludvig Bohlin, Umeå University, for his work on the myocardial tissue velocity quantification method, and BSc. Sandra Gustavsson, Department of Public Health and Clinical medicine, Umeå Universiy for her help with the ultrasound exams. This work was supported by the Swedish research council (VR 2007-3959), the European

Union Regional Development Fund, the Heart foundation of northern Sweden, and the Kempe foundations.

\section{Author details}

${ }^{1}$ Department of Biomedical Engineering - R\&D, Radiation Sciences, Umeå University, Umeå 90185, Sweden. ${ }^{2}$ Centre for Biomedical Engineering and Physics (CMTF), Umeå University, Umeå, Sweden. ${ }^{3}$ Department of Cardiovascular sciences, KU Leuven, Leuven, Belgium. ${ }^{4}$ Heart center and Department of Public Health and Clinical Medicine, Umeå University, Umeå, Sweden.

Received: 14 May 2013 Accepted: 12 September 2013

Published: 16 October 2013

References

1. Sampath S, Kimm JH, Lederman RJ, McVeigh ER: Simultaneous imaging of myocardial motion and chamber blood flow with SPAMM n' EGGS (Spatial Modulation of Magnetization with encoded gradients for gauging speed). J Magn Reson Imaging 2008, 27(4):809-817.

2. Li C, Zhang J, Zhou C, Huang L, Tang H, Rao L: Will simultaneous measurement of E/e' facilitate the noninvasive assessment of ventricular filling pressure in patients with non-valvular atrial fibrillation. Eur J Echocard 2010, 11:296-301. 
3. Lou J, Konofagou EE: Imaging of wall motion coupled with blood flow velocity in the heart and vessels in vivo: A feasibility study. Ultrasound in Med Biol 2011, 37:980-995.

4. Trahey GE, Smith SW, von Ramm OT: Speckle-pattern correlation with lateral aperture translation: Experimental results and implications for spatial compounding. IEEE Trans Ultrason Ferroelectr Freq Control 1986, 32:257-264

5. Bohs LN, Trahey GE: A novel method for angle independent ultrasonic imaging of blood flow and tissue motion. IEEE Trans Biomed Eng 1991, 38:280-286.

6. $\mathrm{Lin} C H$, Lin $M C J$, Sun $Y N$ : Ultrasound motion estimation using a hierarchical feature weighting algorithm. Comp Med Imag Grap 2007, 31:178-190.

7. Mårtensson M, Bjällmark A, Brodin LÅ: Evaluation of tissue Doppler-based velocity and deformation imaging: a phantom study of ultrasound systems. Eur J Echocard 2011, 10:467-476.

8. Jensen JA: Field: A Program for Simulating Ultrasound Systems, Paper presented at the 10th Nordic-Baltic conference on biomedical imaging published in medical \& biological engineering \& computing. Med Biol Eng Comput 1996, 34(1):351-353.

9. Nagueh SF, Middleton K, Kopelen HA, Zoghbi WA, Quiñones MA: Doppler tissue imaging: A noninvasive technique for evaluation of left ventricular relaxation and estimation of filling pressures. J Am Coll Cariol 1997, 30:1527-1533.

10. Lindqvist $P$, Wikström G, Waldenström A: The use of $E / E m$ and the time interval difference of isovolumic relaxation (TIVRT-IVRTm) in estimating left ventricular filling pressures. Eur J Heart Fail 2008, 10:490-497.

11. Szabo T: Diagnostic ultrasound imaging: Inside-out (biomedical engineering). USA: Elsevier Academic Press; 2004

12. Sutherland GR, Hatle L, Claus P, d'Hooge J, Bijnens BH: Doppler Myocardial Imaging - A Textbook. Belgium: BSWK bvba, Scientific Consulting and Publishing- Hasselt; 2006.

doi:10.1186/1475-925X-12-107

Cite this article as: Grönlund et al: Simultaneous quantification of myocardial and blood flow velocities based on duplex mode ultrasound imaging. BioMedical Engineering OnLine 2013 12:107.

\section{Submit your next manuscript to BioMed Central and take full advantage of:}

- Convenient online submission

- Thorough peer review

- No space constraints or color figure charges

- Immediate publication on acceptance

- Inclusion in PubMed, CAS, Scopus and Google Scholar

- Research which is freely available for redistribution

Submit your manuscript at www.biomedcentral.com/submit 